\title{
Exploring the Solid-Liquid Phase Change of an Adapted Dzugutov Model Using Generalized Replica Exchange Method
}

\author{
Qing Lu, ${ }^{\dagger}$ Jaegil Kim, ${ }^{\ddagger} \S$ and John E. Straub* ${ }^{*}$ \\ ${ }^{\dagger}$ Division of Materials Science and Engineering, Boston University, Brookline, Massachusetts 02446, United States \\ ${ }^{\ddagger}$ Department of Chemistry, Boston University, Boston, Massachusetts 02215, United States
}

ABSTRACT: The generalized replica exchange method ( $g$ REM) is applied to simulate a solidliquid phase change in an adapted Dzugutov model system. The idea of $g R E M$ is to incorporate the merit of sampling from a generalized ensemble into the replica exchange paradigm. The generalized ensemble weights are determined from effective temperatures tailored for this system, through an inverse mapping strategy. The ordered and disordered phases are smoothly joined together through a succession of unimodal energy distributions, providing a continuous transformation from an isotropic liquid into an amorphous solid phase under the given condition. The phase change temperature is determined by evaluation of various thermodynamic and structural order parameters. The increasing tendency for icosahedral local order under cooling is quantified using bond order parameters. The ergodic measure is used to demonstrate the advantage of $g$ REM over the traditional temperature-based REM in sampling the phase change region.

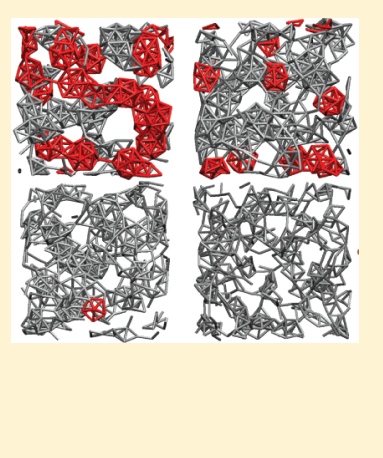

\section{INTRODUCTION}

The replica exchange method (REM) (or parallel tempering) $)^{1,2}$ has been widely used in the computer simulation of diverse complex systems such as proteins, ${ }^{3-8}$ glasses, ${ }^{9-11}$ and atomic clusters, ${ }^{12,13}$ where methods based on sampling the conventional canonical ensemble struggle to attain ergodic sampling over a rugged energy landscape characterized by multiple minima separated by high barriers. ${ }^{14-16}$ In the standard temperature REM ( $t$ REM), a set of statistically independent canonical molecular dynamics (MD) or Monte Carlo (MC) simulations run in parallel at specified temperatures. The coupling of low- and high-temperature replicas via exchanges of configurations allows the low-temperature replicas to escape from trapped regions more easily, facilitating ergodic sampling. ${ }^{13}$ While $t$ REM has proven to be highly effective in equilibrium sampling of stable phase states, the standard $t$ REM struggles to attain its maximum power in the vicinity of a firstorder phase transition. ${ }^{17}$ In moving across a strong phase change, canonical energy distributions are effectively disjointed and characterized by an energy gap corresponding to a latent heat. Since the acceptance probability of replica exchanges is determined by the energy overlap of neighboring replicas, an energy gap between $P_{T<T c}(E)$ and $P_{T>T c}(E)$ around the critical temperature $T_{c}, P_{T}(E)$ being the canonical probability density function (PDF) at the temperature $T$, significantly impairs replica exchanges. The acceptance of replica exchanges for a pair of inverse temperatures, $\beta$ and $\beta^{\prime}$, close to $\beta_{c}=1 / T_{c}$, becomes exponentially suppressed as $A\left(\beta E ; \beta^{\prime} E^{\prime}\right)=$ min$\left[1, \mathrm{e}^{\Delta \beta\left(E^{\prime}-E\right)}\right] \approx \mathrm{e}^{-|\Delta \beta \Delta E|}$, where $\Delta \beta=\beta^{\prime}-\beta$ and $\Delta E$ is the energy gap. The generalized REM $(g R E M)^{18}$ has been developed to restore the full power of replica exchange by incorporating noncanonical ensembles into the replica exchange paradigm. The generalized ensemble sampling weights are determined from tailored effective temperatures through an inverse mapping strategy. Illustrative simulations on a Potts spin system with varying system size and simulation conditions demonstrated a comprehensive sampling for phasecoexistent states with a dramatic acceleration of tunneling transitions. $^{18}$

We apply $g$ REM to explore the phase change properties of an adapted Dzutugov model system. The MD simulation of this simple monatomic system was reported by Elenius and Dzugutov. ${ }^{19}$ Their results demonstrated that upon constantdensity cooling toward a critical point, the isotropic liquid is continuously transformed into a low-density phase with a mesoscopic order similar to that of a smectic liquid crystal. In this work, we test the effectiveness of $g$ REM for achieving enhanced sampling while exploring thermodynamic changes and structural transformations associated with the phase change.

\section{METHODS}

gREM in the Isothermal-Isobaric Ensemble. REM $^{18}$ combines generalized ensembles with replica exchanges in order to restore the full power of replica exchange in the vicinity of a strong phase transition. To handle volume fluctuations in solid-liquid phase change here we extend $g R E M$ to the isothermal-isobaric ensemble. In contrast to the NVT ensemble where the internal energy, $E$, of the system is a natural dynamic variable at the fixed volume $V$, the enthalpy $H$ $=E+P V$, which describes the thermal energy change when a

Special Issue: Macromolecular Systems Understood through Multiscale and Enhanced Sampling Techniques

Received: January 12, 2012

Revised: $\quad$ March 21, 2012

Published: March 27, 2012 
process occurs at constant pressure, $P$, becomes the key dynamical variable in the NPT ensemble.

Around the phase change region, the enthalpy distribution, $P_{\alpha}(H)$, becomes bimodal due to a large energy or volume gap separating two phases, where $\alpha$ denotes a replica index. Sampling unstable or metastable, phase-mixed configurations becomes a rare event due to a large free energy barrier. The fundamental idea of an isothermal-isobaric gREM is to systematically design a set of generalized ensembles, $W_{\alpha}(H)$, so that unstable or metastable enthalpy states corresponding to the phase change region are transformed into stable ones, resulting in a unimodal $P_{\alpha}(H)$. In the most general case, the enthalpy distribution associated with $W_{\alpha}$ is determined as

$$
P_{\alpha}(H)=\Omega(H) W_{\alpha}(H)
$$

where $\Omega(H)$ is the density of states in enthalpy defined by $\int \delta[H(E(r), V)-H] \mathrm{d} r \mathrm{~d} V$. By defining an isobaric entropy, $S(H)=\ln \Omega(H)\left(k_{\mathrm{B}}=1\right)$, analogous to the entropy in the microcanonical ensemble, eq 1 further transforms into $\exp \left\{S(H)-w_{\alpha}(H)\right\}=\exp \left\{-\beta \mathcal{F}_{\alpha}(H)\right\}, \mathcal{F}_{\alpha}(H)$ being the generalized free enthalpy density and $w_{\alpha}$ being the generalized effective potential. The generalized partition function is obtained as $Z_{\alpha}(\beta)=\int \mathrm{d} H \mathrm{e}^{-\beta F_{\alpha}(H)}$.

The key quantity in the isothermal-isobaric gREM is the effective temperature, $T_{\alpha}\left(H ; \lambda_{\alpha}\right)=\left[\partial w_{\alpha} / \partial H\right]^{-1}$, which completely determines the generalized sampling weight up to a constant through the inverse mapping relation

$$
W_{\alpha}(H)=-\ln \int \frac{1}{\tilde{T}_{\alpha}\left(H^{\prime} ; \lambda_{\alpha}\right)} \mathrm{d} H^{\prime}
$$

where $\lambda_{\alpha}$ is a set of parameters characterizing the effective temperature. It is possible to identify conditions for the effective temperature ensuring that the underlying unstable state will be transformed into the stable enthalpy state with a unimodal PDF, $P_{\alpha}(H)$.

A necessary and sufficient condition on $T_{\alpha}\left(H ; \lambda_{\alpha}\right)$ is derived by identifying an extremum, $H_{\alpha}{ }^{*}$, of a generalized free enthalpy density, $\beta \mathcal{F}_{\alpha}(H)=S(H)-w_{\alpha}(H)$,

$$
T_{\alpha}\left(H_{\alpha}{ }^{*} ; \lambda_{\alpha}\right)=T_{S}\left(H_{\alpha}^{*}\right)=T_{\alpha}^{*}
$$

where $T_{\mathrm{S}}(H)=[\partial S / \partial H]^{-1}$ is the statistical temperature in enthalpy analogous to the statistical temperature in the microcanonical ensemble, and $H_{\alpha}{ }^{*}$ is the crossing point between $T_{\alpha}(H)$ and $T_{S}(H)$. By identifying a stability condition

$$
\beta \mathcal{F}_{\alpha}^{\prime \prime}\left(H_{\alpha}{ }^{*}\right)=\left(\gamma_{\mathrm{S}}-\gamma_{\alpha}\right) / T_{\alpha}{ }^{2}
$$

where $\gamma_{\mathrm{s}}=T_{\mathrm{S}}{ }^{\prime}\left(H_{\alpha}{ }^{*}\right), \gamma_{\alpha}=T_{\alpha}{ }^{\prime}\left(H_{\alpha}{ }^{*}\right)$, and the prime denotes differentiation with respect to $H$, we find that a unimodal PDF can arise about the unique crossing point, $H_{\alpha}{ }^{*}$, between $T_{\mathrm{S}}(H)$ and $T_{\alpha}\left(H ; \lambda_{\alpha}\right)$, subject to the condition that $\gamma_{\alpha}\left(H_{\alpha}{ }^{*}\right)<\gamma_{S}\left(H_{\alpha}{ }^{*}\right)$.

In the isothermal-isobaric gREM, exchange between neighboring replicas is performed where replicas represent noncanonical ensembles characterized by the effective temperature $T_{\alpha}\left(H ; \lambda_{\alpha}\right)(\alpha=1,2, \ldots, M)$. As the parameter $\lambda_{\alpha}$ varies, $T_{\alpha}\left(H ; \lambda_{\alpha}\right)$ covers a range of temperature between $T_{1}$ and $T_{M}$, the lowest and highest temperature, respectively. On the basis of the one-to-one correspondence, the sampling weight $W_{\alpha}\left(H, \lambda_{\alpha}\right)$ is completely determined by the inverse mapping in eq 2 .

The simplest parametrization scheme for forming stable crossing points between $T_{\alpha}\left(H ; \lambda_{\alpha}\right)$ and $T_{S}(H)$ is to align linear effective temperatures in parallel with the constant slope, $\gamma$, as

$$
T_{\alpha}\left(H ; \lambda_{\alpha}\right)=\lambda_{\alpha}+\gamma\left(H-H_{0}\right)
$$

the control parameter $\lambda_{\alpha}$ being the $T$-intercept at an arbitrarily chosen $H_{0}$. To form the unique stable crossing point $H_{\alpha}{ }^{*}$ in each replica, $\gamma$ must be less than the minimum slope $\gamma_{S}^{\min }, \gamma_{S}^{\min }=$ $\min \left\{T_{\mathrm{S}}{ }^{\prime}(H)\right\}$ being the minimum slope of $T_{\mathrm{S}}(H)$ for the sampled enthalpy region. Since $T_{S}(H)$ monotonically increases except for the phase change region, in most cases a proper $\gamma$ is easily guessed from the approximate $T_{\mathrm{S}}(H)$ by connecting a few points of $[\tilde{H}(T), T], \tilde{H}(T)$ being an average enthalpy of a short canonical run at $T$. For example, $\gamma$ can be simply chosen as $\gamma=$ $\left(T_{M}-T_{1}\right) /\left(\tilde{H}_{1}-\tilde{H}_{M}\right), T_{1}$ and $T_{M}$ being the lowest and highest temperature, and $\tilde{H}_{\alpha}=\tilde{H}\left(T_{\alpha}\right)$.

Once $\gamma$ is fixed, the dynamic range of $\lambda_{\alpha}$ is determined to cover the interesting temperature range between $T_{1}$ and $T_{M}$ as $\lambda_{1}=T_{1}$ and $\lambda_{M}=T_{M}-\gamma\left(\tilde{H}_{M}-\tilde{H}_{1}\right)$, with $H_{0}=\tilde{H}_{1}$. The first and $M$ th effective temperatures are chosen to cross $\left[\tilde{H}_{1}, T_{1}\right]$ and $\left[\tilde{H}_{M}, T_{M}\right]$, respectively. The intermediate values of $\lambda_{\alpha}(1<\alpha<$ $M)$ are determined by equally dividing the parameter space as

$$
\lambda_{\alpha}=\lambda_{1}+(\alpha-1) \Delta \lambda
$$

and $\Delta \lambda=\left(\lambda_{M}-\lambda_{1}\right) /(M-1)$.

Interestingly, the linear effective temperature of eq 5 produces a generic form of the Tsallis weight ${ }^{20-22}$

$$
W_{\alpha}\left(H ; \lambda_{\alpha}\right) \sim\left[\lambda_{\alpha}+\gamma\left(H-H_{0}\right)\right]^{-1 / \gamma}
$$

Identifying $\gamma$ and $\lambda_{\alpha}$ by $(q-1)$ and $\beta_{\alpha}^{-1}$, respectively, $q$ being the nonextensivity parameter, recovers the original form of the Tsallis weight proposed in nonextensive statistical mechanics. ${ }^{20}$ The $g$ REM with a linear effective temperature of eq 5 is equivalent to the Tsallis-weight-based REM, which has been exploited previously in the form of the generalized parallel tempering (GPT), ${ }^{23} q$-REM, ${ }^{24}$ and Tsallis-REM algorithms. ${ }^{25}$

Detailed simulation protocols of the $g$ REM are defined by the following three steps:

(i) Perform short canonical runs at several temperatures between $T_{1}$ and $T_{M}$ to determine the data set, $\left[\tilde{H}_{\alpha}, T_{\alpha}\right]$. Select a proper $\gamma$ to be less than $\gamma_{S}^{\mathrm{min}}$, and determine $\lambda_{\alpha}$ by employing eq 6 between $\lambda_{1}=T_{1}$ and $\lambda_{M}=T_{M}-\gamma\left(\tilde{H}_{M}-\tilde{H}_{1}\right)$, with $H_{0}=\tilde{H}_{1}$.

(ii) Run the $g$ REM simulation in each replica by making trial moves in configuration space with the acceptance probability

$$
A_{\text {intra }}\left(x \rightarrow x^{\prime}\right)=\min \left[1, \mathrm{e}^{w_{\alpha}(H)-w_{\alpha}\left(H^{\prime}\right)}\right]
$$

Every fixed time step, attempt a replica exchange between neighboring replicas with the acceptance

$$
A_{\text {inter }}\left(\alpha ; x x^{\prime}\right)=\min \left[1, \exp \left(\Delta_{\alpha}\right)\right]
$$

$\Delta_{\alpha}=w_{\alpha+1}\left(H^{\prime}\right)-w_{\alpha+1}(H)+w_{\alpha}(H)-w_{\alpha}\left(H^{\prime}\right)$.

(iii) Once a sufficiently long production run has been performed, calculate the entropy estimate $\tilde{S}(H)$ by joining multiple generalized ensemble runs via the weighted histogram analysis method (WHAM $)^{26}$ or the recently developed statistical temperature weighted histogram analysis method (ST-WHAM). ${ }^{27}$

In the canonical MC runs, the temperatures of the replicas are uniformly distributed between the lowest and highest temperature $T_{1}$ and $T_{M}$. The initial configurations for each replica are prepared by first equilibrating the system at high temperature, and then gradually cooling the system until it reaches the temperature of that replica. In other words, instead of one initial structure for all replicas, a set of initial structures is used according to the temperature of each replica. 
Simple Monatomic Dzugutov Model. We apply gREM to study the solid-liquid phase change properties of a singlecomponent system with an adapted Dzugutov potential (donoted as Z2). ${ }^{19,28}$ The Z2 potential has the form

$$
V(r)=a \frac{\mathrm{e}^{\alpha r}}{r^{3}} \cos \left(2 k_{\mathrm{F}} r\right)+b\left(\frac{\sigma}{r}\right)^{n}+V_{0}
$$

for $r<r_{\mathrm{c}}$ and 0 otherwise. We use the position of the third minimum in the function as our cutoff distance, $r_{\mathrm{c}}$, and $V_{0}$ is defined through the equation $V\left(r_{\mathrm{c}}\right)=0 . V_{0}$ acts to shift the potential so that it vanishes at the third minimum, thus making the function and its first derivative continuous at the cutoff. The potential is plotted in Figure 1 together with the Lennard-Jones

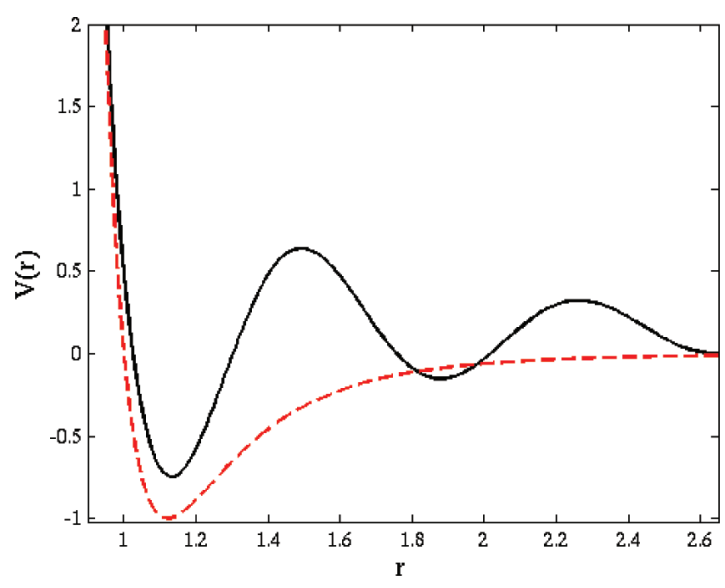

Figure 1. The Z2 potential (refs 22 and 23) used in this study (solid line) compared to the Lennard-Jones potential (dashed line). Plotted after Figure 1 in ref 23.

potential, and the values of the parameters for the potential ${ }^{28,29}$ are given in Table 1 . We use a system of 500 identical particles and set the pressure to be $P=0.40$ for all replicas in order to simulate the NPT ensemble rather than NVT ensemble explored previously for this model system. ${ }^{19}$ Using constant pressure simulation in the vicinity of a phase change, the system is free to transform completely into the state of lowest free energy, which may not be achieved in constant volume simulation. All simulation results are expressed in reduced units of length and energy, which are defined by Lennard-Jones, with the particle mass assumed to be unity. Considering the Lennard-Jones potential as a model potential of argon, and the mass of the argon atom as the unit of mass, the reduced units of length and energy can be estimated as $0.34 \mathrm{~nm}$ and $0.238 \mathrm{kcal} / \mathrm{mol}^{29}$

The $\mathrm{Z} 2$ potential was designed to imitate effective interionic interactions in liquid metals with characteristic Friedel oscillations. $^{30,31}$ The first term in the potential has a form similar to that expected for the effective interaction between metal ions screened by electrons. Friedel oscillations are present with wave vector $2 k_{\mathrm{F}}$, where $k_{\mathrm{F}}$ corresponds to the wave vector at the Fermi level. The second term adds a repulsive interaction that suppresses the oscillations at small $r$. The potential has been found to induce a pronounced local icosahedral ordering of the nearest neighbors due to the design of its short-range attraction, while the repulsion incorporated in the longer-range interaction inhibits bulk packing of icosahedra.

Measuring the Sampling Convergence Using the Ergodic Measure. The energy metric is a convenient quantity to assess the time intervals needed for effective ergodicity to be established by following the dynamics of a system using two distinct initial conditions. ${ }^{32}$ The energy metric $d(t)$ is defined in terms of time averages of energies of individual particles. The time-averaged potential energy of particle $j$ over a trajectory is computed as

$$
e_{j}(t)=\frac{1}{t} \int_{0}^{t} E_{j}(s) \mathrm{d} s
$$

where $E_{j}(s)$ is the energy of particle $j$ at time $s$ on the trajectory. Because the potential energy in this study is pairwise additive, $E_{j}(s)$ is one-half of the sum of all potential-energy terms involving particle $j$. Two independent initial states of the system are chosen, and these are labeled $a$ and $b$. The energy metric, $d(t)$, is given by the square of the energy difference between the energy averages of the particles over the two trajectories, namely,

$$
d(t)=\frac{1}{N} \sum_{j=1}^{N}\left[e_{j a}(t)-e_{j b}(t)\right]^{2}
$$

where the sum runs over all $N$ particles of the system. For an ergodic system, we expect that $\lim _{t \rightarrow \tau}\left[e_{j a}(t)-e_{j b}(t)\right] \rightarrow 0$ for each particle $j$. This is because the time averages for the system reach their equilibrium values in a time $\tau$ independent of any initial condition. In practice, an acceptable estimate for $\tau$ is obtained by determining when $d(\tau) / d(0)=0.01$ is satisfied. $^{32}$

The behavior of $d(t)$ for times greater than a transient time $t_{\mathrm{I}}$ is characterized by a single parameter, $D_{\mathrm{E}}$, the "diffusion constant" associated with the rate of exploration of phase space. The decay of $d(t)$ for $t>t_{\mathrm{I}}$ has been shown to obey the dynamical scaling form

$$
d(t)=d(0) f\left(t D_{\mathrm{E}}\right)
$$

where

$$
f(x)= \begin{cases}1 & x \rightarrow 0 \\ 1 / x & x \gg 1\end{cases}
$$

The reciprocal of $D_{\mathrm{E}}$ is roughly the time required for effective ergodicity to be obtained.

To apply the ergodic measure in the REM, one must compute the measure for each replica separately, because the replicas are at different temperatures and the sampling speeds will, in general, depend on temperature or underlying generalized ensemble. Two independent initial states are given to each replica $\alpha=1, \ldots, M$, and the two trajectories $(a, \alpha)$ and $(b, \alpha)$ are generated. The energy metric for replica $\alpha$ is computed as

$$
d^{\alpha}(t)=\frac{1}{N} \sum_{j=1}^{N}\left[e_{j a}^{\alpha}(t)-e_{j b}^{\alpha}(t)\right]^{2}
$$

\section{Table 1. Values of the Parameters for the Z2 Potential}

$\begin{array}{ccccccccc}a & \alpha & k_{\mathrm{F}} & b & \sigma & V_{0} & n & r_{\mathrm{c}} & \\ 1.04 & 0.33 & 4.139 & 4.2 \times 10^{6} & 0.348 & 14.5 & 2.64488\end{array}$


where $e_{j a}^{\alpha}$ is the time averaged energy of particle $j$, computed using eq 11, and the superscript $\alpha$ indicates replica $\alpha$.

Probing Structure Change Using Pair Distribution Functions. The radial distribution function is computed from the simulation trajectories as

$$
g(r)=\frac{1}{\rho_{0} N}\left\langle\sum_{i=1} \sum_{j \neq i} \delta\left(r-r_{i j}\right)\right\rangle
$$

where $\rho_{0}$ is the bulk density, $r_{i j}$ is the distance between atom $i$ and $j$, and $\langle\cdots\rangle$ denotes an ensemble average.

Let us denote the first minimum of $g(r)$ as $r_{1}$. The coordination number, which is the average number of neighbors, $C N$, up to a distance $r_{1}$, can be computed as ${ }^{33}$

$$
C N=4 \pi \rho_{0} \int_{0}^{r_{c}} r^{2} g(r) \mathrm{d} r
$$

since the area under the first peak of $g(r)$ is proportional to the number of particles in the first coordination shell.

The static structure factor $S(Q)$ is the Fourier transform of the radial distribution function $g(r)^{34,35}$ according to

$$
S(Q)=1+4 \pi \rho_{0} \int_{0}^{L} r^{2}\left(\frac{\sin (Q r)}{Q r}\right)[g(r)-1] \mathrm{d} r
$$

The wave vector $Q$ is defined as $Q=2 \pi k / L$, where $k$ is an integer that ranges from 1 to $N, N$ is the total number of particles, and $L$ is the length of the periodic boundary box. $S(Q)$ provides a measure of the correlation length of the density fluctuations.

Identifying Structural Symmetry through Bond Orientational Order Parameters. Bond orientational order parameters $^{36-38}$ based on spherical harmonics, also known as Steinhardt order parameters, are often used to determine crystal structures in molecular simulations. In computational studies of crystallization from an undercooled liquid, one must distinguish particles that are part of the crystal from those that belong to the liquid. Ideally, such an assignment is based on the local environment of the particles only. One method, which is independent of the specific crystal structure and does not require the definition of a reference frame, is provided by the following algorithm based on spherical harmonics.

The complex vector $q_{l m}(i)$ of particle $i$ is defined as $q_{l m}(i)=$ $1 /\left(N_{b}(i)\right) \sum_{j=1}^{N_{b}(i)} Y_{l m}\left(\mathbf{r}_{i j}\right)$, where, $N_{b}(i)$ is the number of nearest neighbors of particle $i$, the functions $Y_{l m}\left(\mathbf{r}_{i j}\right)$ are the spherical harmonics, and $\mathbf{r}_{i j}$ is the vector from particle $i$ to particle $j$. To make the order parameters invariant with respect to rotations of the reference frame, the third-order invariants are defined as

$$
w_{l}(i)=\sum_{m_{1}+m_{2}+m_{3}=0}\left(\begin{array}{ccc}
l & l & l \\
m_{1} & m_{2} & m_{3}
\end{array}\right) q_{l m_{1}}(i) q_{l m_{2}}(i) q_{l m_{3}}(i)
$$

where the coefficients $(\cdots)$ are the Wigner $3 j$ symbols. We refer to the normalized quantity

$$
\hat{w}_{l} \equiv \frac{w_{l}}{\left(\sum_{m}\left|q_{l m}\right|^{2}\right)^{3 / 2}}
$$

Using this approach, one can determine the type of crystalline structure occurring around each individual particle.

Determining Thermodynamic Quantities Using STWHAM Analysis. We employ a recently developed iterationfree approach to solve the WHAM equations in terms of intensive variables. This numerical approach, the ST-WHAM, ${ }^{27}$ yields the inverse temperature $\beta_{\mathrm{S}}=\partial S / \partial H$ directly from a novel form of the WHAM equations. The statistical temperature is then computed as $T_{\mathrm{S}}=1 / \beta_{\mathrm{S}}$. Thermodynamic quantities including the entropy can be evaluated upon numerical integration of this statistical temperature. Once the entropy is determined, all other canonical thermodynamic properties can be computed, including the specific heat $C_{\mathrm{p}}$, which can be computed as $C_{\mathrm{p}}=T(\partial S / \partial T)_{\mathrm{p}}$.

\section{RESULTS AND DISCUSSION}

Effective Temperatures and Generalized Ensemble Distributions. To determine the dynamic range of $\lambda_{\alpha}$ and the optimal value of $\gamma$, we first performed short canonical MC simulations for $2 \times 10^{4} \mathrm{MC}$ sweeps (MCS), at $T_{1}=0.3$ and $T_{M}$ $=0.6$, which determine $\tilde{H}_{1}=-0.7157$ and $\tilde{H}_{M}=2.0128$. One MCS includes $N$ trial moves, $N$ is also the same as the number of particles, and $M$ is the total number of replicas $(M=31)$. The value of $\gamma$ is computed as $\gamma=\left(T_{M}-T_{1}\right) /\left(\tilde{H}_{1}-\tilde{H}_{M}\right) \approx$ -0.11 . Setting $H_{0}=\tilde{H}_{1}$ in eq 5 , the dynamic range of $\lambda_{\alpha}$, between $\lambda_{1}=T_{1}$ and $\lambda_{M}=T_{M}-\gamma\left(\tilde{H}_{M}-\tilde{H}_{1}\right)$, depends on $\gamma$. For $\gamma=-0.11, \lambda_{1} \approx 0.30$ and $\lambda_{M} \approx 0.72$.

The generalized sampling weights can be determined by eq 7 once the parameters $\gamma$ and $\lambda_{\alpha}$ are known. Replica exchanges were attempted after every replica completed one MCS. Resulting effective temperatures (solid lines) in Figure 2a were chosen to fully span the phase change region. For comparison, we also plot the exact $T_{\mathrm{S}}(H)$, which was determined by the STWHAM. $^{27}$ All relevant parameters in the gREM have been chosen based on short canonical runs at $T_{1}$ and $T_{M}$, and full knowledge of $T_{S}(H)$ is not necessary.
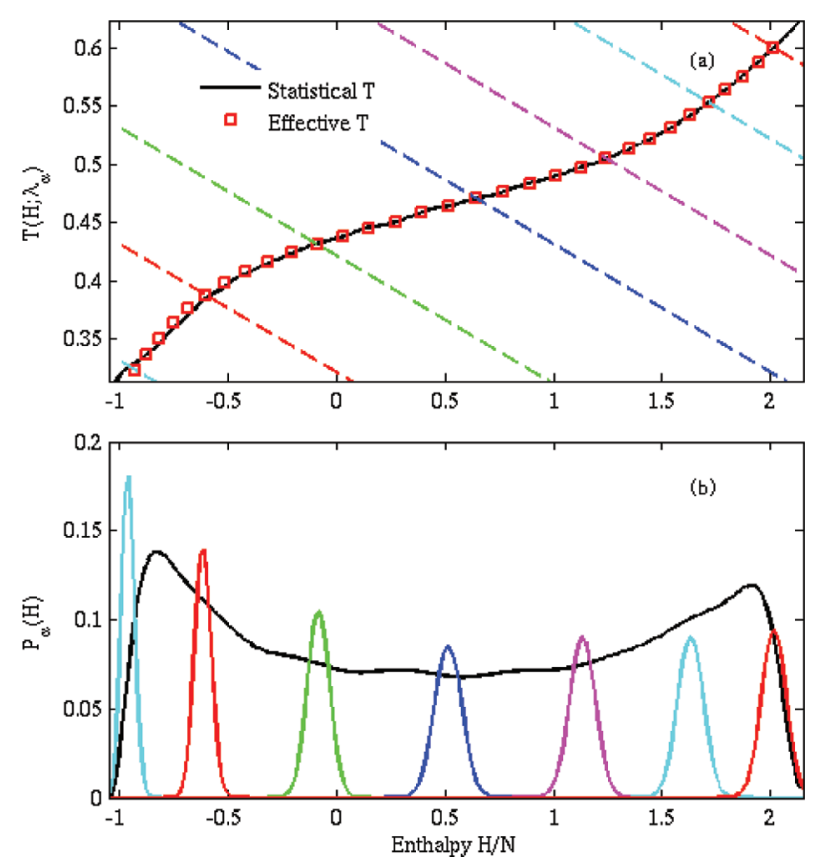

Figure 2. (a) Most probable energy set $\left[H_{\alpha}{ }^{*}, T_{\alpha}{ }^{*}\right]$ (red squares) determined by $g$ REM for $10^{7} \mathrm{MCS}$, and statistical temperature $T_{\mathrm{S}}(H)$ (black solid line). Here $H_{\alpha}{ }^{*}$ is the average enthalpy of replica $\alpha$, and $T_{\alpha} *$ is the effective temperature. The parallel dashed lines represent $T\left(H ; \lambda_{\alpha}\right)$ linear effective temperatures of replica $\alpha$. (b) PDFs $P_{\alpha}(H)$ and superimposed energy distribution function $P_{\mathrm{T}}(H)$ (black line). In both (a) and (b), $\alpha=1,6,11,16,21,27,31$ from left to right. Same colors are applied for the same simulations in both panels. 
Since $T_{\alpha}\left(H ; \lambda_{\alpha}\right)$ was designed to form a unique, stable crossing point, $H_{\alpha}{ }^{*}$, with $T_{\mathrm{S}}(H)$, the resulting PDFs in Figure $2 \mathrm{~b}$ are rapidly localized around a given $H_{\alpha}{ }^{*}$ with a Gaussian shape, and naturally bridge between ordered and disordered phases with unimodal energy distributions across the change region. Since $P_{\alpha}(H)$ is sharply peaked at $H_{\alpha}{ }^{*}, T\left(H_{\alpha}{ }^{*}, \lambda_{\alpha}\right)=$ $T_{S}\left(H_{\alpha}{ }^{*}\right)$, the set of most probable energies, $\left[H_{\alpha}{ }^{*}, T_{\alpha}{ }^{*}\right.$, asymptotically converges toward a locus of $T_{S}(H)$. Indeed, the profile of $\left[H_{\alpha}{ }^{*}, T_{\alpha}{ }^{*}\right]$ shows a perfect coincidence with $T_{\mathrm{S}}(H)$ determined by ST-WHAM, and exactly corresponds to crossing points between $T_{S}(H)$ and $T_{\alpha}\left(H ; \lambda_{\alpha}\right)$ in Figure 2a. For convenience, the most probable energy $H_{\alpha}{ }^{*}$ was approximated by the average energy summed over the $\alpha$ th replica.

For each individual replica, the enthalpy distribution function $P_{\alpha}(H)$ has a single peak around $H_{\alpha}{ }^{*}$ with a Gaussian shape. However, the superimposed enthalpy distribution, $P_{\mathrm{T}}(H)=(1 /$ M) $\sum_{\alpha} P_{\alpha}(H)$, shown in Figure $2 \mathrm{~b}$ as a black curve, displays a bimodal distribution. The locations of the two peaks correspond to the solid state and liquid state, respectively. Despite the existence of the two peaks, $P_{\mathrm{T}}(H)$ is flattened in the entire enthalpy range, which ensures a comprehensive sampling for phase change region.

Sampling Speed Evaluated Using the Ergodic Measure. We have demonstrated the effectiveness of $g R E M$ to achieve comprehensive sampling. In this section we examine the sampling efficiency of gREM compared with traditional $t$ REM using ergodic measure to quantify the sampling speed and rate of convergence of thermodynamic averages.

The energy metric and reciprocal metric are computed using eqs 11 and 12 by following two sets of energy trajectories with two independent initial conditions. As stated earlier, the energy metric for an ergodic system is expected to behave as $d(t) / d(0)$ $=1 / D_{\mathrm{E}} t$ so that the reciprocal metric scales as $d(0) / d(t)=D_{\mathrm{E}} t$. The diffusion constant, $D_{\mathrm{E}}$, measures the sampling speed in phase space. Our results in Figure 3 confirm that the reciprocal

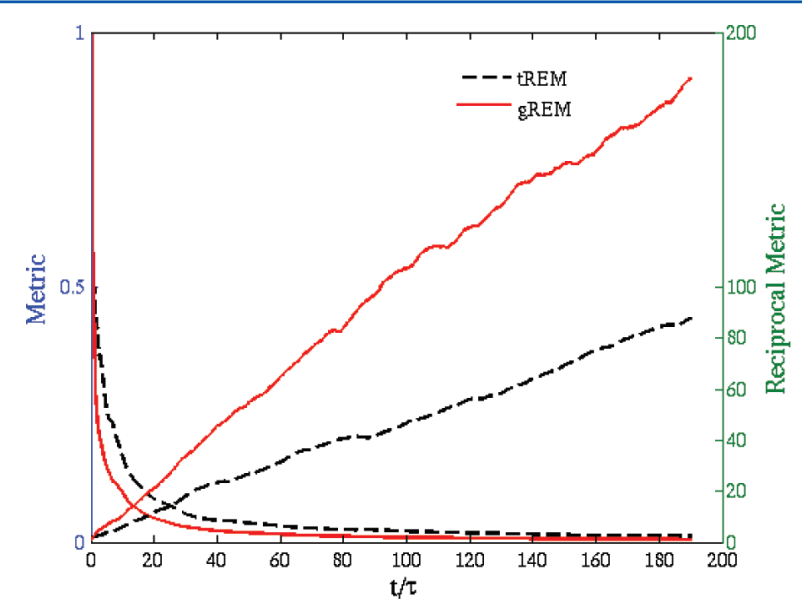

Figure 3. Energy metric $d(t) / d(0)$ and reciprocal metric $d(0) / d(t)$ of $t$ REM (black dash line) and $g$ REM (red solid line) are shown for the 17 th replica. The simulatio time $t$ is scaled by $\tau$, the equilibrium time step, which equals $3 \times 10^{4}$ MCS.

metric is a linear function of time for both $t$ REM and $g$ REM, and the slope of the reciprocal metric of $g R E M$, which gives the diffusion constant $D_{E}$, is nearly 2 times greater than that of $t$ REM.

We computed the diffusion constants $D_{\mathrm{E}}(\alpha)$ of all replicas $(\alpha$ $=1, \ldots, M)$ for $t$ REM and $g$ REM. In $t$ REM, the temperature of $\alpha$ th replica is $T_{\alpha}=T_{1}+(\alpha-1) \Delta T$, where $\Delta T=\left(T_{M}-T_{1}\right) /$ $(M-1), T_{1}$ and $T_{M}$ being the lowest and highest temperature. In $g$ REM, the most probable effective temperature $T_{\alpha}{ }^{*}$ was computed by eq 5 as $T_{\alpha}\left(H ; \lambda_{\alpha}\right)=\lambda_{\alpha}+\gamma\left(H_{\alpha}{ }^{*}-H_{0}\right)$, where the most probable enthalpy $H_{\alpha}{ }^{*}$ was approximated by the average enthalpy summed over the $\alpha$ th replica. As $D_{\mathrm{E}}$ and temperature of each replica are known, we can plot $D_{\mathrm{E}}$ against the temperature at that replica. Figure 4 presents $D_{E}$ of $t$ REM and

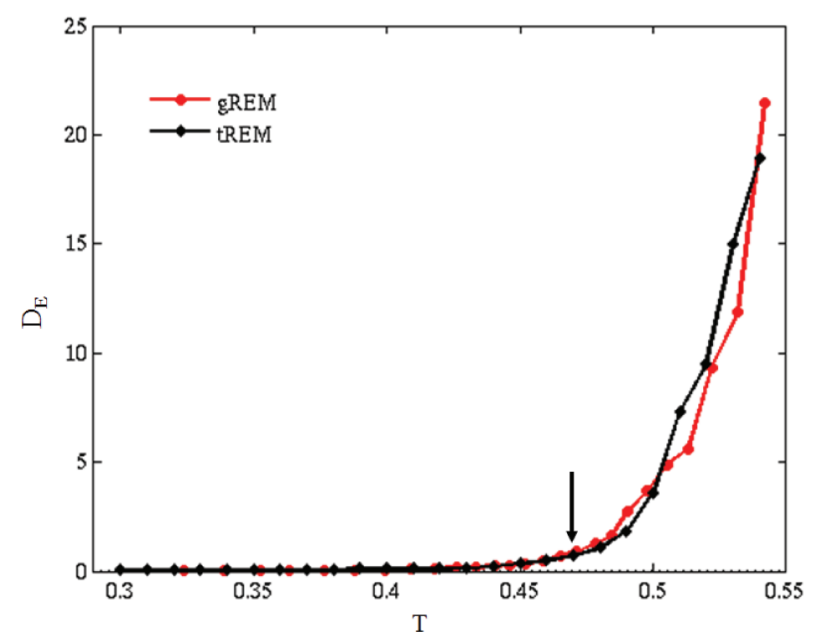

Figure 4. The temperature variation of diffusion constant $D_{\mathrm{E}}$ for both gREM (red) and $t$ REM (black) result. The vertical arrow indicates the melting temperature.

$g R E M$ as a function of temperature within $T=[0.3,0.55]$. Similar results were observed for $g$ REM and $t$ REM. At low temperatures $T=[0.3,0.45], D_{\mathrm{E}}$ stays at a low value when temperature increases. Once temperature goes beyond $T=$ $0.47, D_{\mathrm{E}}$ begins to increase dramatically. There is a 3 orders of magnitude difference between the diffusion constant, $D_{\mathrm{E}}$, of the lowest and highest temperature. This indicates that the system undergoes a phase change from liquid to amorphous solid upon cooling from $T=0.6$ to 0.3 . Note that the reciprocal of $D_{\mathrm{E}}$ in Figure 4 also provides an estimated time scale for each replica to reach effective ergodicity.

Investigation of Liquid-Solid Phase Change. The thermodynamic phase change and phase change temperature were investigated, structurally by calculating the radial distribution function, and thermodynamically by computing the temperature variation of the specific heat.

The radial distribution function $g(r)$ is an appropriate tool to describe the short-range order in a monatomic liquid, providing the probability of finding two atoms in the liquid at a separation $r$. The computed $g(r)$ of the liquid displays a prominent first peak centered at the nearest-neighbor spacing in the crystalline solid indicating the existence of substantial short-range order. The short-range ordering decreases at high temperature due to thermal fluctuation and increases at low temperature. As shown in Figure 5a from the bottom to the top curve, the first peak of $g(r)$ grows taller as temperature decreases. The second and third peaks also become more visible as temperature drops. Meanwhile, the location of each peak remains the same regardless of the temperature. It is worthwhile to point out that even at the lowest temperature $T=0.3, g(r)$ shows characteristics of the liquid state. In the crystalline state, $g(r)$ has an infinite number of sharp peaks whose separations and heights are characteristic of the lattice structure. In the liquid 

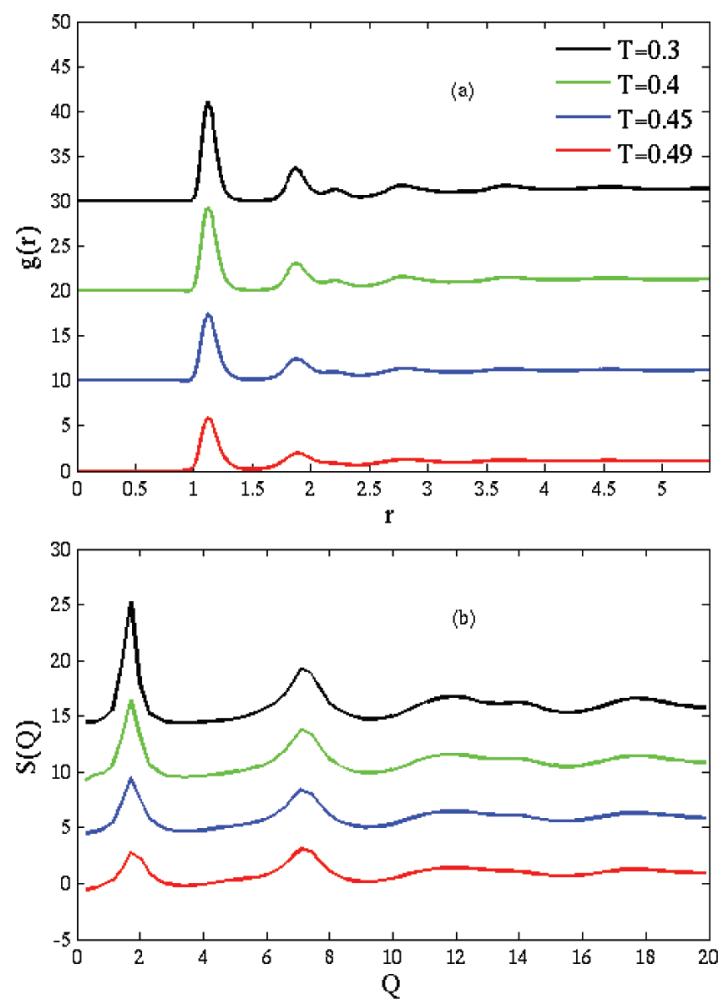

Figure 5. (a) Radial distribution function $g(r)$ and (b) structure factor $S(Q)$ at four different temperatures as noted in (a), where $r$ is the distance and $Q$ is the wavevector. The same color scheme is used for the same temperature in both (a) and (b).

state, the second peak is less prominent and the third peak is hardly visible in $g(r)$, indicating a lack of long-range order. Correlations in particle positions rapidly die out in a liquid, and $g(r)$ approaches unity over a distance of a few particle diameters.

The static structure factor $S(Q)$ can be computed through the Fourier transform of the radial distribution function $g(r)$ by eq 17. Figure $5 \mathrm{~b}$ presents the results of $S(Q)$ showing that at all temperatures $S(Q)$ has a nearest neighbor peak located around $Q \approx 2 \pi$. Below the location of the nearest neighbor peak, there is an additional low- $Q$ prepeak, indicating the formation of the clustering and the resulting residual repulsive interaction between clusters. The presence of the low- $Q$ prepeak in $S(Q)$ at a finite wavevector highlights the presence of an additional characteristic length scale in this system. ${ }^{39}$ Under cooling, the height of the prepeak increases, and the width becomes narrower. However, the similarity of the two curves indicates that we observe a phase change between two similarly structured phases.

The coordination number $(\mathrm{CN})$ measures the average number of nearest neighbors, and reflects the degree of short-range order in a system. Figure 6 shows the temperature variation of the coordination number, which shows a gradual decrease as the temperature increases. At low temperature $T=$ $0.3, \mathrm{CN} \approx 10$, indicating that on average each particle has 10 nearest neighbors. The short-range ordering is characteristic of the solid state, but as discussed earlier, the steady decay of $g(r)$ indicates the lack of long-range ordering. The nature of the low temperature state is determined to be an amorphous solid state. As temperature increases, $\mathrm{CN}$ drops dramatically, reflecting that

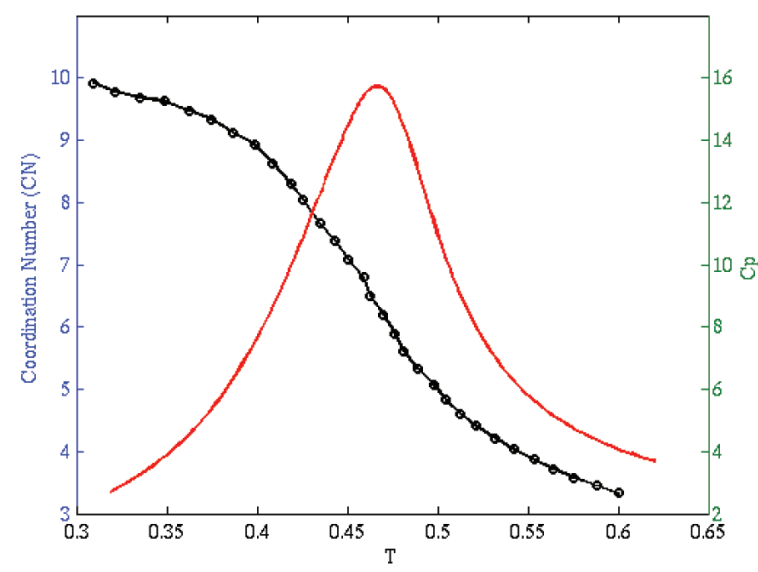

Figure 6. Temperature variation of coordination number $(\mathrm{CN})$ and specific heat $\left(C_{\mathrm{p}}\right)$ near the phase change temperature in black and red lines, respectively.

the system is melting around a characteristic temperature around $T=0.47$.

The temperature variation of the specific heat at constant pressure, $C_{p}$, is also shown in Figure 6 , and displays a pronounced maximum at $T=0.47$. The appearance of excess specific heat under cooling is an indication of the development of bonded structure, and a common precursor of percolation. $^{30,31}$ The maximum of $C_{\mathrm{p}}$, as well as the low- $Q$ prepeak of $S(Q)$, is commonly observed in the context of gelation in colloidal systems. ${ }^{39,40}$ The maximum temperature $T_{\mathrm{m}}=0.47$ is in agreement with the phase change temperature derived from the coordination number.

Assessing Icosahedral Cluster Formation Using Bond Order Parameters. Bond-orientational order parameters were computed to determine the nature of the local symmetry underlying the local order observed at low temperatures. The four bond order parameters $q_{4}, q_{6}, \hat{w}_{4}$, and $\hat{w}_{6}$ are generally sufficient to identify crystal structures reflecting face-centered cubic (fcc), body-centered cubic (bcc), haxagonal close-packed (hcp), and icosahedra (Ih) structure. We focus on icosahedral local ordering, which is a pronounced feature of this adpated Dzugutov model system. Local icosahedral ordering is characterized by large negative values of $\hat{w}_{6}$, up to $\hat{w}_{6}=$ -0.1675 for a perfect icosahedron. ${ }^{36}$

The value of $\hat{w}_{6}$ for each individual particle is computed using eq 18 . If $\hat{w}_{6} \leq-0.165$, the particle is counted as icosahedrally ordered, and its nearest neighbors within a cutoff radius of 1.5 are also included in the icosahedral cluster. In Figure 7 we present the computed temperature variation in the number of icosahedrally coordinated particles, $N_{\text {Ih }}$, scaled by system size $N$. The value of $N_{\text {Ih }} / N$ remains almost temperature independent for $T>0.5$, and grows rapidly with cooling below $T=0.47$, which is also the location of the maximum in the specific heat, $C_{\mathrm{p}}$. This phase change temperature is coincident with the bond percolation threshold, where the bonds are defined between pairs of atoms that are nearest neighbors in icosahedral clusters. At low temperature $T=0.3$, nearly $50 \%$ of the particles belong to icosahedral clusters.

Figure 8 shows the distributions of $\hat{w}_{6}$ at four temperatures across the phase change range. The reference value for ideal icosehedral ordering is plotted as a red dashed line. At $T_{1}=0.3$, $\hat{w}_{6}$ has a sharp and narrow peak located close to the reference value, indicating that a large percentage of particles have icosahedral local ordering. At $T_{2}=0.4$, the peak of the $\hat{w}_{6}$ 


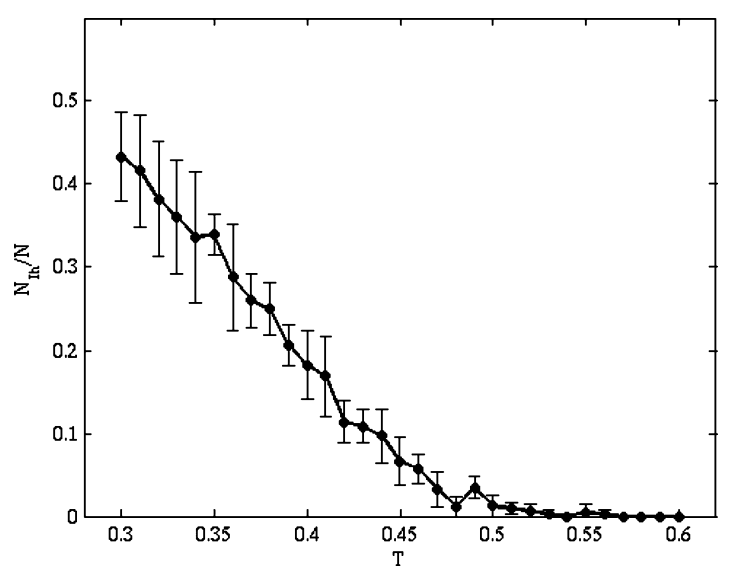

Figure 7. The number of particles of local icosahedra structure $N_{\text {Ih }}$ scaled by the total number of particle in the system $N$ as a function of temperature between $T=[0.3,0.6]$. The error bars are defined as standard deviation from 9 sets of independent results.

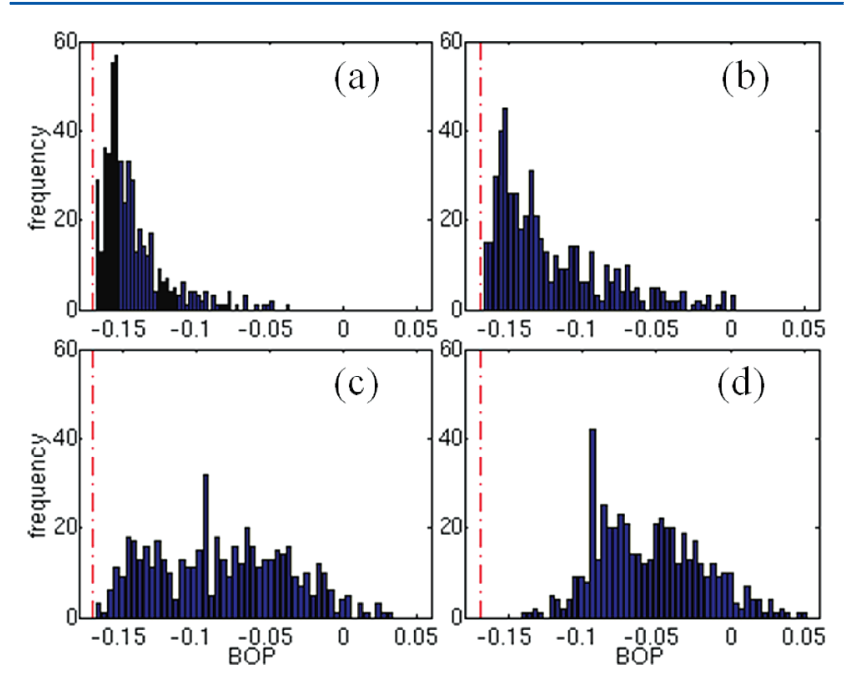

Figure 8. The distribution of bond order parameter $\hat{w}_{6}$ at four temperatures $T=0.3,0.4,0.5,0.6,(a, b, c, d$, respectively). The red dashed line is the reference value of $\hat{w}_{6}$ for the ideal icosahedral symmetry.

distribution remains close to the reference value, but the distribution becomes wider. Increasing the temperature further to $T_{3}=0.5$ and $T_{4}=0.6$, the icosahedral symmetry is mostly absent.

Insight into the structral transformation from solid to liquid is provided by direct inspection of the atomic configurations. Figure 9 depicts four configurations at $T_{1}=0.3$ to $T_{4}=0.6$. At $T_{1}$ the connected icosahedral clusters occupy a large percentage of the volume. At $T_{2}=0.4$, the number of icosahedral clusters is diminished, and the icosahedra are scattered and disjoint. When $T_{3}=0.5$, the number of icosahedral cluster is further diminished. As temperature increases to $T_{4}=0.5$, the icosahedral ordering is found to be absent in the high temperature liquid state. These results are consistent with earlier observations regarding the evolution of icosahedral order in this system under cooling (see Figure 7).

\section{CONCLUSION}

The applicability of the gREM has been demonstrated in an adapted Dzugutov model system. By combining optimally parametrized generalized ensemble sampling with the replica

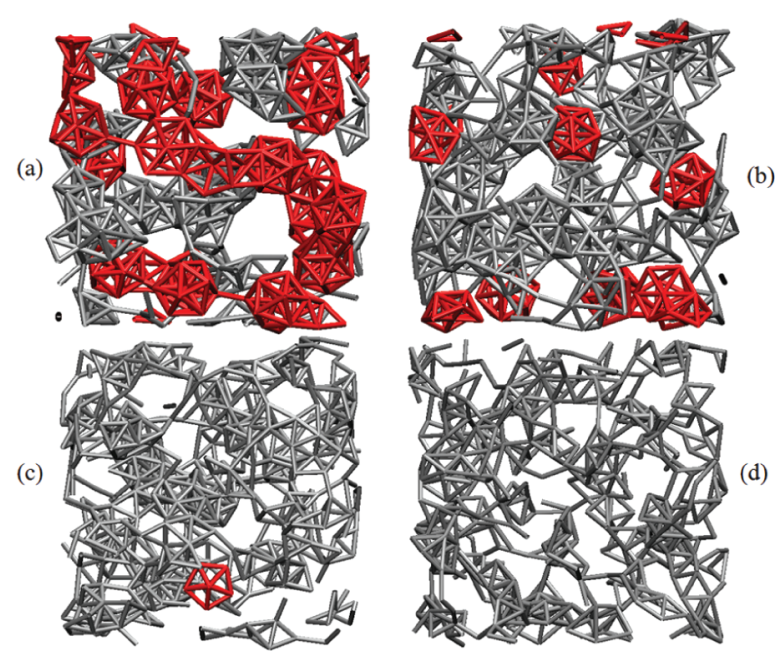

Figure 9. Four respresentative configurations at four temperatures $T=$ $0.3,0.4,0.5,0.6$, (a, b, c, d, respectively). The icosehedral clusters are marked in red, and the nonicosahedral particles are marked in gray. The cutoff distance for a bond is 1.5 . Note that the size of each plot does not represent the real volume at that temperature.

exchange protocol, the $g$ REM provides effective sampling across the phase change region through successive unimodal energy distributions. The ergodic measure comparison between $g$ REM and $t$ REM reveals the advantage of $g$ REM in achieving effective conformational sampling across the phase change region. The diffusion constant computed by the ergodic measure shows a 3 orders of magnitude difference between the highest and lowest temperature state, indicating that the system goes from a liquid to amorphous solid upon cooling. The temperature evolution of the radial distribution function and structure factor demonstrates that the low temperature state is not a crystalline solid, but rather an amorphous solid possessing short-range icosahedral order. The phase change temperature was determined to be $T=0.47$ through evaluation of the temperature variation of the coordination number and specific heat through the solid-liquid phase change. The growth of icosahedral clustering under cooling is observed and quantified with the aid of bond order parameters.

Our results indicate that the solid states of lowest free energy for the range of pressures studied have an amorphous character and lack long-range translational symmetry characteristic of an ordered solid. The adapted Dzugatov potential imposes characteristic length-scales that will be reflected in a structured liquid or solid, including the core particle size (dictated by the repulsive potential), the position of the first minimum, and the distance between the first and second potential minimum (or intervening maxima) resulting from the potential oscillations. The solid state is characterized by the formation of icosahedral clusters, the size of which is largely determined by the core particle size and position of the first potential minima. These "superparticles" are then found to arrange themselves on a lattice geometry, where the distance between lattice sites will be determined by the interactions between superparticles. Depending on the relation between the positions of second and third minima and maxima and the overall icosahedral superparticle size, the interactions may be stabilized or result in frustration. With the parametrization of the adapted Dzugatov model studied in this work, which was tailored to prevent crystallization, $^{28}$ these competing length scales lead to frustration that disrupts long-range order. Optimization of 
these interactions and parameters could potentially lead to a thermodynamically stable solid state exhibiting long-range translational order, as well as a stronger and more cooperative transition between the liquid and solid states.

The absence of a substantial difference in the measured rate of conformational sampling for $g$ REM as opposed to $t$ REM results from the modest nature of the solid-liquid phase change in this system and the absence of a substantial energy gap. As a result, the distribution of states in energy or enthalpy is relatively continuous for temperature replicas as well as for the generalized energy distributions. The gradual nature of this phase change is in part due to the amorphous nature of the observed solid state, which lacks long-range translational order. More dramatic enhancements in sampling using the gREM protocol can be expected for systems demonstrating stronger phase transitions with a more substantial energy gap and a range of unstable states in the canonical ensemble.

\section{AUTHOR INFORMATION}

\section{Corresponding Author}

*E-mail: straub@bu.edu.

\section{Present Address}

${ }^{\S}$ Broad Institute of MIT and Harvard, Cambridge, MA, 02142. Notes

The authors declare no competing financial interest.

\section{ACKNOWLEDGMENTS}

We are grateful to the National Science Foundation (Grant No. CHE-1114676) for the generous support of our research. We are thankful to Edyta Malolepsza for her technical support.

\section{REFERENCES}

(1) Geyer, C. J.; Thompson, A. J. Am. Stat. Assoc. 1995, 90, 909-920.

(2) Hukushima, K.; Nemoto, K. J. Phys. Sco. Jpn. 1996, 65, 16041608 .

(3) Sugita, Y.; Okamoto, Y. Chem. Phys. Lett. 1999, 314, 141-151.

(4) Zhou, R.; Berne, B. J. Proc. Natl. Acad. Sci. U.S.A. 2002, 99, 12777-12782.

(5) Garcia, A. E.; Onuchic, J. N. Proc. Natl. Acad. Sci. U.S.A. 2003, 100, 13898-13903.

(6) Paschek, D.; Gnanakarnan, S.; Garcia, A. E. Proc. Natl. Acad. Sci. U.S.A. 2005, 102, 6765-6770.

(7) Hansmann, U. H. E. Chem. Phys. Lett. 1997, 281, 140-150.

(8) Zheng, W.; Andrec, M.; Gallicchio, E.; Levy, R. M. Proc. Natl. Acad. Sci. U.S.A. 2007, 104, 15340-15345.

(9) Yamamoto, R.; Kob, W. Phys. Rev. E 2000, 61, 5473-5476.

(10) Flenner, E.; Szamel, G. Phys. Rev. E 2006, 73, 061505.

(11) Berg, B. A.; Celik, T. Phys. Rev. Lett. 1992, 69, 2292-2295.

(12) Frantsuzov, P. A.; Mandelshtam, V. A. Phys. Rev. E 2005, 72, 037102.

(13) Poulain, P.; Calvo, F.; Antoine, R.; Broyer, M.; Dugourd, P. Phys. Rev. E 2006, 73, 056704.

(14) Berne, B. J.; Straub, J. E. Curr. Opin. Struct. Biol. 1997, 7, 181189.

(15) Mitsutake, A.; Sugita, Y.; Okamoto, Y. Biopolymers 2001, 60, 96-123.

(16) Li, H.; Li, G.; Berg, B. A.; Yang, W. J. Chem. Phys. 2006, 125, 144902.

(17) Earl, D. J.; Deem, M. W. Phys. Chem. Chem. Phys. 2005, 7, 3910-3916.

(18) Kim, J.; Keyes, T.; Straub, J. E. J. Chem. Phys. 2010, 132, 224107.

(19) Elenius, M.; Dzugutov, M. J. Chem. Phys. 2009, 131, 104502.

(20) Tsallis, C. J. Stat. Phys. 1988, 52, 479-487.

(21) Andricioaei, I.; Straub, J. E. Phys. Rev. E 1996, 53, R3055R3058.
(22) Andricioaei, I.; Straub, J. E. J. Chem. Phys. 1997, 107, 91179124.

(23) Whitfield, T. W.; Bu, L.; Straub, J. E. Physica A 2002, 305, 157171.

(24) Jang, S.; Shin, S.; Park, Y. Phys. Rev. Lett. 2003, 91, 058305.

(25) Kim, J.; Straub, J. E. J. Chem. Phys. 2009, 107, 144114.

(26) Ferrenberg, A. M.; Swenden, R. H. Phys. Rev. Lett. 1989, 63, $1195-1198$.

(27) Kim, J.; Keyes, T.; Straub, J. E. J. Chem. Phys. 2011, 135, 061103.

(28) Doye, J. P. K; Wales, D. J.; Zetterling, F. H. M.; Dzugutov, M. J. Chem. Phys. 2003, 118, 2792-2799.

(29) Elenius, M.; Oppelstrup, T.; Dzugutov, M. J. Chem. Phys. 2010, $133,174502$.

(30) Moriarty, J. A.; Widom, M. Phys. Rev. B 1997, 56, 7905-7917.

(31) Ashcroft, N. W.; Mermin, N. D. Solid State Physics; Saunders Book Company: Collingwood, ON, Canada, 1976.

(32) Thirumalai, D.; Mountain, R. D. Phys. Rev. A 1990, 42, 45744587.

(33) Moore, E. B.; Molinero, V. J. Chem. Phys. 2009, 130, 244505.

(34) Salacuse, J. J.; Denton, A. R.; Egelstaff, P. A. Phys. Rev. E 1996, $53,2382-2389$.

(35) Herrera, J. N.; Cummings, P. T.; Ruiz-Estrada, H. Mol. Phys. 1999, 96, 835-847.

(36) Steinhardt, P. J.; Nelson, D. R.; Ronchetti, M. Phys. Rev. B 1983, $28,784-805$.

(37) Y Wang, S. T.; Dellago, C. J. Chem. Phys. 2005, 122, 214722.

(38) Lechner, W.; Dellago, C. J. Chem. Phys. 2008, 129, 114707.

(39) Sciortino, F.; Tartaglia, P.; Zaccarelli, E. J. Phys. Chem. B 2005, 109, 21942-21953.

(40) Bianchi, E.; Tartaglia, P.; Nave, E. L.; Sciortino, F. J. Phys. Chem. B 2007, 111, 11765-11769. 